\title{
On Semiopen Sets and Semicontinuous Functions in Intuitionistic Fuzzy Topological Spaces
}

\author{
Shyamal Debnath \\ Department of Mathematics, Tripura University,Suryamaninagar Agartala-799022, India
}

\begin{abstract}
The purpose of this paper is to introduce "semiopen sets" in intuitionistic fuzzy topological spaces. After giving the fundamental definitions and necessary examples we introduce the definitions of intuitionistic fuzzy semicontinuity, intuitionistic fuzzy semicompactness, intuitionistic fuzzy semiconnectedness and studied several preservations properties and some characterizations theorems. We see that every intuitionistic fuzzy open set is intuitionistic fuzzy semiopen and every intuitionistic fuzzy continuous function is intuitionistic fuzzy semicontinuous.
\end{abstract}

Key words: Intuitionistic fuzzy topology, intuitionistic fuzzy semiopen sets, intuitionistic fuzzy semicontinuous functions, intuitionistic fuzzy semi $C_{5}$-connectedness, intuitionistic fuzzy semicompactness.

\section{Introduction}

After the introduction of the concept of fuzzy sets by Zadeh [10] several researches were conducted on the generalizations of the notion of fuzzy set. The idea of intuitionistic fuzzy set was first published by Atanassov [1] and D.Coker [6], [7] introduced the notion of intuitionistic fuzzy topological spaces, intuitionistic fuzzy continuity, compactness, connectedness and some other related concepts. I.M.Hanafy [9] introduced the concept of completely continuous functions in intuitionistic fuzzy topological spaces. In this paper we introduce and study the concept of semiopen sets and semicontinuous functions in intuitionistic fuzzy topological spaces.

\section{Preliminaries:}

Throughout this section, we shall present the fundamental definitions and results of intuitionistic fuzzy sets and intuitionistic fuzzy topology as given by Atanassov[2] and Coker[7].

Definition 2.1[2] Let $X$ be a nonempty fixed set. An intuitionistic fuzzy set (IFS for short) A is an object having the form, $A=\left\{\left\langle x, \mu_{A}(x), \gamma_{A}(x)\right\rangle: x \in X\right\}$, where the functions $\mu_{A}: X \rightarrow I$ and $\gamma_{A}: X \rightarrow I$ denote the degree of membership ( namely $\mu_{A}(x)$ ) and the degree of nonmembership ( namely $\gamma_{A}(x)$ ) of each element $\mathrm{x} \in X$ to the set $\mathrm{A}$, and $0 \leq \mu_{A}(x)+\gamma_{A}(x) \leq 1$ for each $x \in X$.

For the sake of simplicity, we shall use the symbol $A=\left\langle x, \mu_{A}, \gamma_{A}\right\rangle$ for the IFS $A=$ $\left\{\left\langle x, \mu_{A}(x), \gamma_{A}(x)\right\rangle: x \in X\right\}$ $\mu A x, x \in X\}$.

Obviously, every fuzzy set A on a nonempty set $\mathrm{X}$ is an IFS having the form, $A=\left\{\left\langle x, \mu_{A}(x), 1-\right.\right.$

Definition 2.2[2] Let $X$ be a nonempty set, and let the IFSs A and B be in the form $A=\left\{\left\langle x, \mu_{A}(x), \gamma_{A}(x)\right\rangle\right.$ : $x \in X\}, B=\left\{\left\langle x, \mu_{B}(x), \gamma_{B}(x)\right\rangle: x \in X\right\}$ and let $\left\{A_{j}: \mathrm{j} \in J\right\}$ be an arbitrary family of IFSs in X. Then
(a)
$\mathrm{A} \subseteq B$ iff $\mu_{A}(x) \leq \mu_{B}(x)$ and $\gamma_{A}(x) \geq \gamma_{B}(x)$ for all $x \in X$
(b) $\bar{A}=\left\{\left\langle x, \gamma_{A}(x), \mu_{A}(x)\right\rangle: x \in X\right\}$;
(c) $\quad 1_{\sim}=\{\langle x, 1,0\rangle: x \in X\}$ and $0_{\sim}=\{\langle x, 0,1\rangle: x \in X\}$;
(d) $\cap \cap A_{j}=\left\{\left\langle x, \wedge \mu_{A_{j}}(x), \vee \gamma_{A_{j}}(x)\right\rangle: x \in X\right\}$;
(e) $\quad \cup A_{j}=\left\{\left\langle x, \vee \mu_{A_{j}}(x), \wedge \gamma_{A_{j}}(x)\right\rangle: x \in X\right\}$;
(f) $\quad[] \mathrm{A}=\left\{\left\langle x, \mu_{A}(x), 1-\mu_{A}(x)\right\rangle: x \in X\right\}$;
(g) $\quad<>A=\left\{\left\langle x, 1-\gamma_{A}(x), \gamma_{A}(x)\right\rangle: x \in X\right\}$;
(h) $\quad \overline{\bar{A}}_{=} A, \overline{1}_{\sim}=0_{\sim}$ and $\overline{0}_{\sim}=1_{\sim}$;
(i) $\overline{A \cup B}=\bar{A} \cap \bar{B}, \overline{A \cap B}=\bar{A} \cup \bar{B}$;

Definition 2.3[7] Let $\mathrm{X}$ and $\mathrm{Y}$ be two nonempty sets and $f: X \rightarrow Y$ be a function.

(i) If $B=\left\{\left\langle y, \mu_{B}(y), \gamma_{B}(y)\right\rangle: y \in Y\right\}$ is an IFS in $Y$, then the preimage of $B$ under $f$ is denoted and defined by $f^{-1}(B)=\left\{\left\langle x, f^{-1}\left(\mu_{B}\right)(x), f^{-1}\left(\gamma_{B}\right)(x)\right\rangle: x \in X\right\}$.

(ii) If $A=\left\{\left\langle x, \lambda_{A}(x), v_{A}(x)\right\rangle: x \in X\right\}$ is an IFS in $X$, then the image of $A$ under $f$ is denoted and defined by $f(A)=\left\{\left\langle y, f\left(\lambda_{A}\right)(y), f_{-}\left(v_{A}\right)(y)\right\rangle: y \in Y\right\}$,

where $f_{-}\left(v_{A}\right)=1-f\left(1-v_{A}\right)$.

In (i),(ii), since $\mu_{B}, \gamma_{B}, \lambda_{A}, v_{A}$ are fuzzy sets, we explain that 
and

$$
f^{-1}\left(\mu_{B}\right)(x)=\mu_{B}(f(x)),
$$

$$
f\left(\lambda_{A}\right)(y)=\left\{\begin{array}{cc}
\sup \lambda_{A}(x) & \text { if } f^{-1}(y) \neq \varnothing \\
0 & \text { otherwise }
\end{array}\right.
$$

Definition 2.4[7] Let $A, A_{i}(i \in J)$ be IFSs in $X$ and $B, B_{j}(j \in K)$ IFSs in $Y$ and $f: X \rightarrow Y$ be a function. Then

(i) $f^{-1}\left(\cup B_{j}\right)=\cup f^{-1}\left(B_{j}\right)$;

(ii) $f^{-1}\left(\cap B_{j}\right)=\cap f^{-1}\left(B_{j}\right)$;

(iii) $f^{-1}\left(1_{\sim}\right)=1_{\sim} ; f^{-1}\left(0_{\sim}\right)=0_{\sim}$;

(iv) $f^{-1}\left(\overline{B)}=\overline{f^{-1}(B)}\right.$;

(v) $f\left(\cup A_{i}\right)=\cup f\left(A_{i}\right)$;

Definition 2.5[7] An intuitionistic fuzzy topology (IFT, for short) on a nonempty set X is a family $\tau$ of IFSs in $\mathrm{X}$ satisfying the following axioms:
(i) $0_{\sim}, 1_{\sim} \in \tau$;
(ii) $A_{1} \cap A_{2} \in \tau$ for any $A_{1}, A_{2} \in \tau$
(iii) $\cup A_{j} \in \tau$ for any $\left\{A_{j}: j \in J\right\} \subseteq \tau$.

In this case the pair $(X, \tau)$ is called an intuitionistic fuzzy topological space (IFTS, for short) and each IFS in $\tau$ is known as an intuitionistic fuzzy open set (IFOS, for short) in $X$. The complement $\bar{A}$ of IFOS $A$ in $\operatorname{IFTS}(X, \tau)$ is called an intuitionistic fuzzy closed set (IFCS) in $X$.

Definition 2.6[7] Let $(X, \tau)$ be an IFTS and $A=\left\langle x, \mu_{A}, \gamma_{A}\right\rangle$ be an IFS in X. The the intuitionistic fuzzy closure and fuzzy interior of $A$ are defined by

$$
\begin{aligned}
& \operatorname{cl}(A)=\cap\{K: K \text { is an IFCS in } X \text { and } A \subseteq K\} \text { and } \\
& \text { int }(A)=\cup\{G: G \text { is an IFOS in } X \text { and } G \subseteq A\}
\end{aligned}
$$

Definition 2.7[7, 9] Let $(X, \tau)$ and $(Y, \emptyset)$ be two IFTSs and $f: X \rightarrow Y$ a function. Then

(i) $f$ is fuzzy continuous iff the preimage of each IFOS in $Y$ is an IFOS in $X$.

(ii) $f$ is fuzzy completely continuous iff the preimage of each IFOS in $Y$ is an IFROS in $X$.

Definition 2.8[9] Let $f: X \rightarrow Y$ be a function. The graph $g: X \rightarrow X \times Y$ of $f$ is defined by

$$
g(x)=(x, f(x)), \forall x \in X .
$$

Lemma 2.9[9] Let $g: X \rightarrow X \times Y$ be the graph of a function $f: X \rightarrow Y$. If $A$ is an IFS of $X$ and $B$ is an IFS of $Y$, then $g^{-1}(A \times B)(x)=\left(A \cap f^{-1}(B)(x)\right.$.

\section{Intuitionistic fuzzy semiopen sets:}

Definition 3.1 Let $A$ be an IFS of IFTS $(X, \tau)$, then $A$ is said to be (i) an intuitionistic fuzzy semiopen (IFSO) set of $X$ if there exists a IFO set $B \in \tau$ such that $B \leq A \leq \operatorname{cl}(B)$,

and (ii) an intuitionistic fuzzy semiclosed (IFSC) set of $X$ if there exists a IFC set $B$ such that $\operatorname{int}(B) \leq A \leq B$.

It can be easily shown that, closure of an IFO (IFC) set of $(X, \tau)$ is IFSO (IFSC) set.

Remark 3.2 It is obvious that every IFO (IFC) set in an IFTS $(X, \tau)$ is IFSO (IFSC) but the converse is not true.

Example 3.3 Let $X=[a, b]$, consider the IFSs on $\mathrm{X}$ are

$$
\begin{gathered}
A=\{(a, 0.5,0.2),(b, 0.5,0.4)\} ; B=\{(a, 0.4,0.5) ;(b, 0.6,0.3)\} ; \\
C=\{(a, 0.5,0.2),(b, 0.6,0.3)\} ; D=\{((a, 0.4,0.5),(b, 0.5,0.4)\} ; \\
\mathrm{E}=\{(a, 0.6,0.1),(b, 0.7,0.2)\} ; F=\{((a, 0.1,0.5),(b, 0.2,0.6)\} ; \\
\quad \text { Then } \tau=\left\{0_{\sim}, 1_{\sim}, A, B, C, D\right\} \text { is a IFT on } X .
\end{gathered}
$$

Also we see that $A \leq E \leq \operatorname{cl}(A)=1_{\sim}$ and $0_{\sim}=\operatorname{int}(\bar{D}) \leq F \leq \bar{D}$.

Thus $E$ is IFSO set but not IFO of $(X, \tau)$ and $F$ is IFSC set but not IFC of $(X, \tau)$.

Theorem 3.4 Let $(X, \tau)$ be an IFTS. Then the following are equivalent:

(i) $A$ is IFSC set

(ii) $\bar{A}$ is IFSO set

(iii) int $\operatorname{cl}(A) \leq A$

(iv) $\operatorname{cl} \operatorname{int}(\bar{A}) \geq \bar{A}$

Proof:

(i) $\Rightarrow$ (ii) By definition there exists a closed set $B$ in $(X, \tau)$ such that $\operatorname{int}(B) \leq A \leq B$, this implies, $\overline{\operatorname{int}(B)} \geq$ $\bar{A} \geq \bar{B}$, using proposition 3.15 of D.Coker[7], we get, $\bar{B} \leq \bar{A} \leq \operatorname{cl}(\bar{B})$ where $\bar{B}$ is an IFO set in $(X, \tau)$. Therefore, $\bar{A}$ is IFSO in $(X, \tau)$.

(ii) $\Rightarrow$ (i) Similar 
(i) $\Rightarrow$ (iii) By definition there exists a IFC set $B$ in $(X, \tau)$ such that $\operatorname{int}(B) \leq A \leq B$ and hence using proposition 3.16 of D. Coker[7], we get, $\operatorname{int}(B) \leq A \leq \operatorname{cl}(A) \leq B$. Since $\operatorname{int}(B)$ is the largest open set contained in $B$, we have int $\operatorname{cl}(A) \leq \operatorname{int}(B) \leq A$ i.e, int $\operatorname{cl}(A) \leq A$.

(iii) $\Rightarrow($ i) This follows by taking $B=\operatorname{cl}(A)$ i.e, $\operatorname{int}(B) \leq A$. Also we know that $A \leq \operatorname{cl}(A)=B$ and hence $\operatorname{int}(B) \leq A \leq B$, as $B=\operatorname{cl}(A)$ is a closed set, therefore $A$ is IFSC.

(ii) $\Rightarrow$ (iv) can similarly be proved.

Theorem 3.5 Union of a finite number of IFSO sets is a IFSO set and intersection of a finite number of IFSC sets is a IFSC set.

Proof:

$1^{\text {st }}$ part Let $A_{1}, A_{2}, \ldots \ldots \ldots, A_{n}$ be IFSO sets of $(X, \tau)$ then their exist IFO sets $B_{1}, B_{2}, \ldots \ldots \ldots B_{n}$ of $(X, \tau)$ such that $B_{i} \leq A_{i} \leq \operatorname{cl}\left(B_{i}\right), i=1(2) n$. Generalizing the idea of proposition 3.16, D.Coker[7] we get,

$$
\cup B_{i} \leq \cup A_{i} \leq \cup \operatorname{cl}\left(B_{i}\right)=\operatorname{cl}\left(\cup B_{i}\right)
$$

Also $\cup B_{i} \in \tau$, Hence $\cup A_{i}$ is IFSO.

$\underline{2^{\text {nd }} \text { part }}$ Similar.

Proposition 3.6 Let $(X, \tau)$ be an IFTS and let $B=\left\{\left\langle x, \mu_{B}(x), \gamma_{B}(x)\right\rangle: x \in X\right\}$ be IFSO sets in $X$, then $\mu_{B}=\left\{\left\langle x, \mu_{B}(x)\right\rangle: x \in X\right\}$ is semiopen in $\left(X, \tau_{1}\right)$ and $\gamma_{B}=\left\{\left\langle x, \gamma_{B}(x)\right\rangle: x \in X\right\}$ is semiclosed in $\left(X, \tau_{2}\right)$, where $\tau_{1}=\left\{\mu_{G}: G \in \tau\right\}$ and $\tau_{2}=\left\{1-\gamma_{G}: G \in \tau\right\}$ are FTS on X in Chang's sense.

Proof: Given $B$ is IFSO, then there exists IFO set $A$ such that $A \subseteq B \subseteq \operatorname{cl}(A)$. Let $A=\left\langle x, \mu_{A}, \gamma_{A}\right\rangle$ and suppose that the family of IFC sets containing $A$ are indexed by $\left\{\left\langle x, \gamma_{G_{i}}, \mu_{G_{i}}\right\rangle: i \in J\right\}$. Then we have, $\operatorname{Cl}(A)=$ $\left\langle x, \wedge \gamma_{G_{i}}, \vee \mu_{G_{i}}\right\rangle$ and $\mu_{A} \leq \gamma_{G_{i}}, \gamma_{A} \geq \mu_{G_{i}}$ for each $i \in J$. By definition of IFSO sets, we get

$\left\langle x, \mu_{A}, \gamma_{A}\right\rangle \subseteq\left\langle x, \mu_{B}, \gamma_{B}\right\rangle \subseteq\left\langle x, \gamma_{G_{i}}, \mu_{G_{i}}\right\rangle$, each $i \in J$

i.e, $\mu_{A} \leq \mu_{B} \leq \gamma_{G_{i}}$ and $\gamma_{A} \geq \gamma_{B} \geq \mu_{G_{i}}$, each $i \in J$

i.e, $\mu_{A} \leq \mu_{B} \leq\left(1-\mu_{G_{i}}\right)$ and $\left(1-\gamma_{G_{i}}\right) \leq \gamma_{B} \leq \gamma_{A}$, each $i \in J$

i.e, $\mu_{A} \leq \mu_{B} \leq \operatorname{cl}\left(\mu_{A}\right)$ and $\operatorname{int}\left(\gamma_{A}\right) \leq \gamma_{B} \leq \gamma_{A}$

i.e, $\mu_{B}=\left\{\left\langle x, \mu_{B}(x)\right\rangle: x \in X\right\}$ is semiopen in $\left(X, \tau_{1}\right)$ and $\gamma_{B}=\left\{\left\langle x, \gamma_{B}(x)\right\rangle: x \in X\right\}$ is semiclosed in $(X$,

$\left.\tau_{2}\right)$.

Example 3.7 Any FTS $\left(X, \tau_{0}\right)$ in the sense of Chang is obviously an IFTS in the form $\tau=\left\{A: \mu_{A} \in \tau_{0}\right\}$ whenever we identify a fuzzy set in $X$ whose membership function is $\mu_{A}$ with its counter part $A=\left\langle x, \mu_{A}, 1-\right.$ $\left.\mu_{A}\right\rangle$. If $\mu_{B}$ is fuzzy semiopen in $\left(X, \tau_{0}\right)$ then $B=\left\langle x, \mu_{B}, 1-\mu_{B}\right\rangle$ is IFSO in $(X, \tau)$.

Since $\mu_{B}$ is semi open in $\left(X, \tau_{0}\right)$, there exists a open set in $\left(X, \tau_{0}\right)$ such that

$$
\begin{gathered}
\mu_{A} \leq \mu_{B} \leq \operatorname{cl}\left(\mu_{A}\right) \\
\text { i.e } 1-\mu_{A} \geq 1-\mu_{B} \geq 1-\operatorname{cl}\left(\mu_{A}\right)=\operatorname{int}\left(1-\mu_{A}\right), \\
\text { i.e } \operatorname{int}\left(1-\mu_{A}\right) \leq 1-\mu_{B} \leq 1-\mu_{A}
\end{gathered}
$$

Therefore $\left(1-\mu_{B}\right)$ is semi closed in $\left(X, \tau_{0}\right)$.

Thus we conclude that $A=\left\langle x, \mu_{A}, 1-\mu_{A}\right\rangle \leq B=\left\langle x, \mu_{B}, 1-\mu_{B}\right\rangle \leq C l(A)$.

Hence $B$ is IFSO in $(X, \tau)$.

\section{Intuitionistic fuzzy semicontinuous functions:}

Definition 4.1 Let $(X, \tau)$ and $(Y, \varnothing)$ be two IFTSs and $f: X \rightarrow Y$ a function. Then $f$ is said to be intuitionistic fuzzy semicontinuous (IFSCn) iff the preimage of each IFO set in $\emptyset$ is an IFSO in $\tau$.

Remark 4.2 Every intuitionistic fuzzy continuous function is intuitionistic fuzzy semicontinuous but the converse is not true.

Example 4.3 Let $X=[a, b]$, consider the IF sets on $X$ are $A=\{(a, 0.5,0.2),(b, 0.5,0.4)\} ; \quad B=\{(a, 0.5,0.2),(b, 0.5,0.2)\}$. Then $\tau=\left\{0_{\sim}, 1_{\sim}, A\right\}$ is a IFT on $X$. Let us consider a function $f:(X, \tau) \rightarrow(X, \tau)$ defined by $f(a)=f(b)=a$, then $f$ is IFS continuous but it is not IF continuous, because $f^{-1}(A)=B \notin(X, \tau)$.

Definition 4.4 Let $(X, \tau)$ and $(Y, \varnothing)$ be two IFTSs and $f: X \rightarrow Y$ a function. Then $f$ is said to be intuitionistic fuzzy semiopen mapping if the image of each IFO set in $\tau$ is an IFSO set in $\emptyset$.

It is obvious that every IF open mapping [7] is IF semiopen mapping.

Example 4.5 Let $\left(X, \tau_{0}\right)$ and $\left(Y, \emptyset_{0}\right)$ be two FTSs in the sense of Chang.

(a) If $f: X \rightarrow Y$ is fuzzy semicontinuous in the usual sense, then in this case, $f$ is IFS continuous. Here we consider the IFTs on $X$ and $Y$ are as follows:

$\tau=\left\{\left\langle x, \mu_{G}, 1-\mu_{G}\right\rangle: \mu_{G} \in \tau_{0}\right\}$ and $\varnothing=\left\{\left\langle y, \lambda_{H}, 1-\lambda_{H}\right\rangle: \lambda_{H} \in \emptyset_{0}\right\}$.

In this case we have, for each $\left\langle y, \lambda_{H}, 1-\lambda_{H}\right\rangle \in \emptyset, \lambda_{H} \in \emptyset_{0}$ 
On Semiopen Sets And Semicontinuous Functions In Intuitionistic Fuzzy Topological Spaces $\overline{f^{-1}\left(\left\langle y, \lambda_{H}, 1-\lambda_{H}\right\rangle\right)=\left\langle x, f^{-1}\left(\lambda_{H}\right), f^{-1}\left(1-\lambda_{H}\right)\right\rangle=\left\langle x, f^{-1}\left(\lambda_{H}\right), 1-f^{-1}\left(\lambda_{H}\right)\right\rangle \text { is IF semiopen set in } \tau \text {. As }}$ $\lambda_{H} \in \emptyset_{0}$ and $\mathrm{f}$ is fuzzy semiopen, therefore $f^{-1}\left(\lambda_{H}\right)$ is fuzzy semiopen in $\left(X, \tau_{0}\right)$ and $1-f^{-1}\left(\lambda_{H}\right)$ is fuzzy semiclosed in $\left(X, \tau_{0}\right)$.

(b) Let $f: X \rightarrow Y$ be a fuzzy semiopen function in the usual sense. Then $f$ is also IF semiopen function in the sense of above definition.

Theorem 4.6 A function $f:(X, \tau) \rightarrow(Y, \emptyset)$ is IFSCn iff $f^{-1}(B)$ is an IFSC set in $X$, for each IFC set $B$ in $Y$. Proof: $\mathbf{1}^{\text {st }}$ part

Let $f$ is IFSCn and also let $B$ be any IF closed set in $Y$, then $\bar{B}$ is an IFO set in $Y$. By definition of IFSCn function, $f^{-1}(\bar{B})$ is IFSO in $(X, \tau)$ i.e $\overline{f^{-1}(B)}$ is IFSO in $(X, \tau)$ i.e $f^{-1}(B)$ is IFSC in $(X, \tau)$.

$2^{\text {nd }}$ part similar

Theorem 4.7 If $f$ is IFSCn function and $g$ is IF continuous then $g$ of $f$ is IFS continuous.

Proof: obvious.

Theorem 4.8 Let $f:(X, \tau) \rightarrow(Y, \varnothing)$ be a function and $g: X \rightarrow X \times Y$, be the graph of the function $f$, then $f$ is IFS continuous if $g$ is so.

Proof: Let $B$ be any IFO set in $Y$, then $f^{-1}(B)=f^{-1}\left(1_{\sim} \times B\right)=1 \sim f^{-1}(B)=g^{-1}\left(1_{\sim} \times B\right)$. Since $B$ is IFO in $Y, 1_{\sim} \times B$ is IFO in $X \times Y$. Also, the fact that $g$ is IFSC implies that $g^{-1}\left(1_{\sim} \times B\right)$ is IFSO in $X$. Hence $f^{-1}(B)$ is IFSO in $X$ and so $f$ is IFS continuous.

Definition 4.9 Let $(X, \tau)$ be an IFTS and $A$ an IFS in $X$.

(a) If a family $\left\{\left\langle x, \mu_{G_{i}}, \gamma_{G_{i}}\right\rangle: i \in J\right\}$ of IFSO sets in $X$ satisfies the condition $A \subseteq \bigcup\left\{\left\langle x, \mu_{G_{i}}, \gamma_{G_{i}}\right\rangle: i \in J\right\}$, then it is called a fuzzy semi open cover of $A$. A finite subfamily of the fuzzy semi open cover $\left\{\left\langle x, \mu_{G_{i}}, \gamma_{G_{i}}\right\rangle: i \in J\right\}$ of $A$, which is also a fuzzy semi open cover of $A$, is called a finite subcover of $\left\{\left\langle x, \mu_{G_{i}}, \gamma_{G_{i}}\right\rangle: i \in J\right\}$.

(b) An IFS $A=\left\langle x, \mu_{A}, \gamma_{A}\right\rangle$ in an IFTS $(X, \tau)$ is called IF semi compact iff every IF semi open cover of A has a finite subcover.

Theorem 4.10 Let $f:(X, \tau) \rightarrow(Y, \emptyset)$ be an IF semicontinuous function. If $A$ is IF semicompact in $(X, \tau)$ then $f(A)$ is IF compact in $(Y, \varnothing)$.

Proof: Let $\mathcal{B}=\left\{G_{i}: i \in J\right\}$, where $G_{i}=\left\{\left\langle y, \mu_{G_{i}}, \gamma_{G_{i}}\right\rangle: i \in J\right\}$ is a IF open cover of $f(A)$. Then $\mathcal{A}=\left\{f^{-1}\left(G_{i}\right): i \in\right.$ $J\}$ is a IF semi open cover of $A$. Since $A$ is IF semi compact, there exists a finite subcover of $\mathcal{A}$ i.e there exists $G_{i},(i=1,2, \ldots, n)$ such that

$A \subseteq \bigcup_{i=1}^{n} f^{-1}\left(G_{i}\right)$. Hence $f(A) \subseteq f\left(\bigcup_{i=1}^{n} f^{-1}\left(G_{i}\right)\right)=\bigcup_{i=1}^{n} f\left(f^{-1}\left(G_{i}\right)\right) \subseteq \bigcup_{i=1}^{n}\left(G_{i}\right)$.

Therefore, $f(A)$ is also IF compact.

Definition 4.11 An IFTS $(X, \tau)$ is said to be IF semi $C_{5}$-connected if the only IF sets which are both IFSO and IFSC are $0_{\sim}$ and $1_{\sim}$

$(X, \tau)$ is said to be IF semi $C_{5}$-disconnected if it is not IF semi $C_{5}$-connected.

Theorem 4.12 Let $f:(X, \tau) \rightarrow(Y, \emptyset)$ be an IF semicontinuous surjection. If $(X, \tau)$ is fuzzy semi $C_{5}$-connected, then $(Y, \emptyset)$ is fuzzy $C_{5}$-connected.

Proof: On the contrary, suppose that $(Y, \varnothing)$ is fuzzy $C_{5}$-disconnected. Then there exists an IF open and closed set $G$ such that $G \neq 1_{\sim}$ and $G \neq 0_{\sim}$. Since $f$ is IF semicontinuous, $f^{-1}(G)$ is both an IFSO and IFSC set. The equalities $f^{-1}(G)=1_{\sim}$ or $f^{-1}(G)=0_{\sim}$ cannot hold. [Because, otherwise we have $G=f\left(f^{-1}(G)\right)=f\left(1_{\sim}\right)=$ $1_{\sim}$ and $\left.G=f\left(f^{-1}(G)\right)=f\left(0_{\sim}\right)=0_{\sim}\right]$ Hence $(Y, \emptyset)$ is $\operatorname{IF} C_{5}$-connected.

\section{References}

[1] K. Atanassov: Intuitionistic fuzzy sets, VII ITKR's Session, Sofia,1983.(In Bulgarian)

[2] K. Atanassov: Intuitionistic fuzzy sets, Fuzzy Sets and Systems 20(1986), 87-96.

[3] K.K.Azad: On fuzzy semicontinuity, fuzzy almost continuity and fuzzy weakly continuity, J.Math.Anal.Appl.82(1981),14-32.

[4] C.L.Chang: Fuzzy topological spaces, J.Math.Anal.Appl.24(1968),182-190.

[5] A.K.Chaudhuri and P.Das, Fuzzy connected sets in fuzzy topological spaces, Fuzzy Sets and Systems 49(1992), $223-229$.

[6] D.Coker and A.H.Es: On fuzzy compactness in intuitionistic fuzzy topological spaces, J. Fuzzy Math. 3(1995), 899-909.

[7] D.Coker: An introduction to intuitionistic fuzzy topological spaces, Fuzzy Sets and Systems 88(1997), 81-89.

[8] H.Gurcay, D.Coker and A.H.Es: On fuzzy continuity in intuitionistic fuzzy topological spaces, J.Fuzzy Math. 5(1997),365-378.

[9] I.M.Hanafy: Completely continuous functions in intuitionistic fuzzy topological spaces, Czechoslovak Mathematical Journal, 53(128) (2003), 793-803.

[10] L.A.Zadeh, Fuzzy sets, Inform. And Control 8(1965)338-353. 